\title{
Genetic influence on methadone treatment outcomes in patients undergoing methadone maintenance treatment for opioid addiction: a pilot study
}

\author{
This article was published in the following Dove Press journal: \\ Neuropsychiatric Disease and Treatment \\ 19 August 2014 \\ Number of times this article has been viewed
}

\author{
Zainab Samaan ${ }^{1-4}$ \\ Monica Bawor 3,4 \\ Brittany B Dennis ${ }^{2,3}$ \\ Carolyn Plater ${ }^{5}$ \\ Michael Varenbut ${ }^{5}$ \\ Jeffrey Daiter ${ }^{5}$ \\ Andrew Worster ${ }^{5,6}$ \\ David C Marsh ${ }^{5,7}$ \\ Charlie $\operatorname{Tan}^{8}$ \\ Dipika Desai ${ }^{3}$ \\ Lehana Thabane $e^{2,9,10}$ \\ Guillaume Pare" \\ 'Department of Psychiatry \\ and Behavioural Neurosciences, \\ ${ }^{2}$ Department of Clinical Epidemiology \\ and Biostatistics, ${ }^{3}$ Population Genomics \\ Program, Chanchlani Research Centre, \\ ${ }^{4}$ MiNDS Neuroscience Program, \\ McMaster University, Hamilton, Ontario, \\ Canada; ${ }^{5}$ Ontario Addiction Treatment \\ Centres, Richmond Hill, Ontario, \\ Canada; ${ }^{6}$ Department of Medicine, \\ McMaster University, Hamilton, Ontario, \\ Canada; ${ }^{7}$ Northern Ontario School \\ of Medicine, Laurentian University, \\ Sudbury, Ontario, Canada; ${ }^{8}$ Michael \\ G. DeGroote School of Medicine, \\ McMaster University, ${ }^{9}$ Biostatistics \\ Unit, Centre for Evaluation of \\ Medicine, ${ }^{10}$ System Linked Research \\ Unit, "Department of Pathology \\ and Molecular Medicine, McMaster \\ University, Hamilton, Ontario, Canada
}

Correspondence: Zainab Samaan Mood Disorders Program, St Joseph's Healthcare Hamilton, 100 West 5 th St, Hamilton, ON, L8N 3K7, Canada $\mathrm{Tel}+\mid 905522$ III55 ext 36372

Fax +l 9055756029

Email samaanz@mcmaster.ca
Introduction: Treatment of opioid addiction with methadone is effective; however, it is known to produce interindividual variability. This may be influenced in part by genetic variants, which can increase the initial risk of developing opioid addiction as well as explain differences in response to treatment. This pilot study aimed to assess the feasibility of conducting a full-scale genetic analysis to identify genes that predict methadone treatment outcomes in this population.

Methods: This was a cross-sectional observational study of patients admitted to a methadone maintenance treatment program for opioid addiction. We obtained demographic and clinical characteristics in addition to blood and urine samples, for the assessment of treatment outcomes.

Results: The recruitment process yielded 252 patients, representing a $20 \%$ recruitment rate. We conducted genetic testing based on a $99.6 \%$ rate of provision of DNA samples. The average retention in treatment was 3.4 years, and $>50 \%$ of the participants reported psychiatric and medical comorbidities. BDNF rs6265 and DRD2 rs1799978 were the common single nucleotide polymorphisms (SNPs) selected for the feasibility study.

Discussion: This study met our predetermined feasibility criteria; recruitment, response rates, and genetic testing were feasible; treatment duration was sufficient for follow up; and the prevalence of comorbid conditions indicated the need for reliable psychiatric and chronic pain measures. The study strengths included effective collaboration with clinics and the generalizability of sample population. Key learning points show the need for assessment of treatment outcomes on multiple domains, implementation of follow up, and the development of standardized training for the study clinical staff.

Keywords: genetics, substitute opioid therapy, treatment response, risk factors

\section{Introduction}

Opioid addiction is a serious chronic psychiatric disorder with detrimental consequences for individuals and society. Risk of infection, death, psychiatric and polysubstance use disorders, adverse social consequences, and criminal activities sustaining major societal costs are rampant among this patient population. ${ }^{1-9}$ The use of prescribed opioids has reached alarming proportions ${ }^{10}$ among the North American population, and the number of prescription opioid-related deaths $(11,499)$ has surpassed that of heroin and cocaine combined. ${ }^{11}$ Canada reports the world's second largest opioid analgesic consumption per capita ${ }^{12}$ and, in Ontario, there are over 35,000 registered methadone patients in addiction treatment centers. ${ }^{13}$ 
The treatment of opioid addiction with methadone maintenance treatment (MMT) is effective but carries significant risks ${ }^{14-16}$ and demonstrates interindividual variability in the clinical response to methadone. ${ }^{17-20}$ This variability is thought to be influenced, in part, by genetic variants, which account for $43 \%-70 \%$ of the risk of opioid addiction. ${ }^{21}$ A search of the literature revealed gaps in knowledge with respect to the role of genetics in methadone treatment. Studies aimed at assessing the effectiveness of MMT have varied greatly and have had several definitions for treatment response, inconsistent outcome measures, and short follow-up duration, leading to the inability to reach valid conclusions. ${ }^{14,22}$ Furthermore, studies investigating the genetic effect on MMT were also inconclusive, largely due to small sample size, short follow-up duration, and limited selection of candidate genes, as seen in a recent review by Dennis et al. ${ }^{23}$ Despite the common use of methadone, the efficacy of such treatment and the choice of an effective yet safe dose continue to be clinical challenges.

The Genes of Opioid Addiction (GENOA) pilot study was designed to identify the genes that are associated with methadone treatment outcomes. The primary objective of this pilot study is to determine the overall feasibility of the GENOA study by assessing the following outcomes:

1. Recruitment rate across the four methadone clinic sites

2. Response rate for the questionnaire, measured as the percentage of subjects that respond to the questions appropriately

3. Feasibility of completing genetic and laboratory data analysis, including the percentage of participants that provide blood samples for genetic screening

4. Retention rate, based on duration between treatment initiation and study entry

5. Rates of substance use, and medical and psychiatric comorbidity among MMT patients.

We also sought to evaluate the demand and utilization of resources, including laboratory availability and the time to complete interviews, data collection, and subsequent analyses, as well as any costs associated with the study. Lastly, we aimed to identify the data management challenges and locate problems with missing information and data heterogeneity.

\section{Methods}

This was a cross-sectional observational study of patients admitted to MMT for the treatment of opioid addiction at four clinical sites of the Ontario Addiction Treatment Centres (OATC), between June and December 2011. We obtained ethics approval from the Hamilton Integrated Research Ethics Board (project number 11-056).

\section{Setting and participants}

The study included a cohort of individuals on MMT, recruited sequentially from OATC clinics. Clinical services were available to all participants, as per usual clinical standards; these included medical examination and laboratory tests, urine testing for illicit substances, supervised daily methadone doses, and addiction counseling. For this pilot study, patients with opioid dependency receiving MMT were enrolled, consisting of men and women, age $\geq 18$ years who are able to provide written informed consent. The exclusion criteria were English language barriers and refusal to provide urine samples.

\section{Data collection and instruments}

Case report forms (CRFs) included demographic variables, such as age, sex, height, weight, employment, ethnicity, and marital status. We also included questions about clinical history that included methadone dose, duration, and age of onset of opioid abuse, physical and psychiatric comorbidity, prescribed and nonprescribed medications, illicit substance use, and family history of addiction/mental health.

\section{Feasibility criteria}

In an effort to ensure realistic recruitment and data collection strategies for the full investigation, we chose to assess the feasibility of investigating the association between genetic variants and methadone treatment outcomes based on a recruitment rate of at least $20 \%$ of all eligible subjects (based in turn on the target sample size and total clinic population) and a $90 \%$ participation rate for provision of blood samples for genetic testing. We also wished to evaluate the availability of resources at community sites (eg, interview space, freezers, centrifuge, and shipping) and the quality of extracted DNA samples. Treatment response and duration were also assessed to help us estimate the sample size needed for a feasible duration of follow up.

\section{Statistical analysis}

The patient demographics were summarized using descriptive summary measures and expressed as mean (standard deviation) or as median (range) for continuous variables (percentage for categorical variables). We performed all analyses using STATA 11 (StataCorp LP, College Station, TX, USA).

\section{Laboratory analysis}

DNA was extracted from whole blood, using standard protocol on a QIAsymphony Automated Nucleic Acid Extraction System (Qiagen, Venlo, the Netherlands). We genotyped 
two common single nucleotide polymorphisms (SNPs) (BDNF rs6265 and DRD2 rs1799978) on Applied Biosystems ${ }^{\circledR}$ ViiA $^{\text {TM }} 7$ Real Time PCR System (Life Technologies Corp, Carlsbad, CA, USA) with Applied Biosystems TaqMan Genotyping Master Mix (Life Technologies Corp). The genotype call rate was $97.7 \%$ and $99.2 \%$ for $B D N F$ rs 6265 and DRD2 rs1799978, respectively. Additionally, clinical staff performed qualitative and semiquantitative urine analysis using the iMDx ${ }^{\mathrm{TM}}$ Analyzer and Prep Assay (NOVX Systems Inc, Richmond Hill, ON, Canada) weekly at the OATC clinical sites throughout the study period, as part of routine clinical care.

\section{Results}

\section{Participant flow}

Four OATC clinics participated in the GENOA pilot study, with a total of approximately 1,300 subjects attending treatment at any given time. During the 6-month period, 260 patients were recruited (Figure 1).

\section{Patient demographics}

Our sample included 252 subjects (150 men and 102 women) with a mean age of 36.9 (SD 10.4) years. The study participants' characteristics are presented in Table 1. The average duration on methadone was 3.4 years. Methadone dose showed large variability, from as low as $2 \mathrm{mg}$ /day to as high as $555 \mathrm{mg} /$ day, in keeping with expected interindividual variation. There was also a substantial family history of substance abuse, with $60 \%$ of participants reporting having one or two parents with addiction disorders. Almost $50 \%$ of participants also reported comorbid psychiatric disorders, with the most common being depression $(32.9 \%)$, and $57.9 \%$ of the population reported a medical comorbidity, with chronic pain being the most common condition (25.4\%).

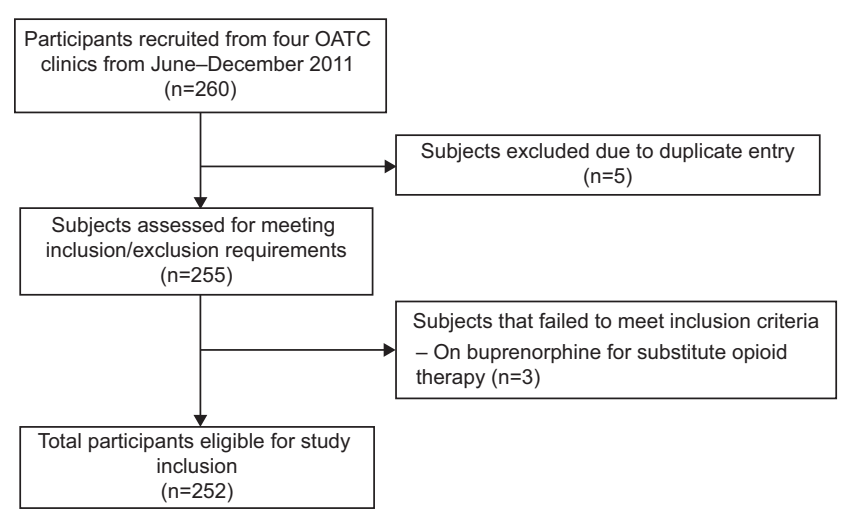

Figure I Flow diagram for participants included in the study. Abbreviation: OATC, Ontario Addiction Treatment Centres.
Table I Demographics of the GENOA study sample

\begin{tabular}{|c|c|}
\hline Variables $(n=252)$ & Values \\
\hline Age in years, mean (SD) & $36.9(10.3)$ \\
\hline Age of opioid onset in years, mean (SD) & $23.2(9.2)$ \\
\hline Methadone use duration in months, mean (SD) & $40.7(42.6)$ \\
\hline Methadone dose in mg/day, median (range) & $80.0(2-555)$ \\
\hline Treatment response, mean (SD) & $81.5(23.2)$ \\
\hline BMI, mean (SD) & $26.8(6.4)$ \\
\hline European ethnicity, \% & 84.9 \\
\hline Currently married/common law, \% & 38.9 \\
\hline Currently employed, \% & 29.8 \\
\hline Current tobacco smoking, \% & 88.9 \\
\hline Current alcohol use, \% & 31.1 \\
\hline \multicolumn{2}{|l|}{ Family history of addiction } \\
\hline Parents, \% & 59.5 \\
\hline Siblings, \% & 41.3 \\
\hline Comorbid psychiatric disorders, $\%$ & 48.8 \\
\hline Depression, \% & 32.9 \\
\hline Bipolar, \% & 8.7 \\
\hline Schizophrenia, \% & 1.6 \\
\hline Anxiety, \% & 27.0 \\
\hline Personality, \% & 4.8 \\
\hline Other, \% & 14.3 \\
\hline Comorbid medical disorders, \% & 42.1 \\
\hline HIV, \% & 0.4 \\
\hline Hepatitis, \% & 24.2 \\
\hline Liver disease, \% & 6.3 \\
\hline Chronic pain, \% & 25.4 \\
\hline Epilepsy, \% & 2.0 \\
\hline Other, \% & 25.8 \\
\hline \multicolumn{2}{|l|}{ Past 12 months self-reported drug use } \\
\hline Heroin, \% & I7. \\
\hline OxyContin, \% & 44.4 \\
\hline Cannabis, \% & 59.8 \\
\hline Stimulants, \% & 48.2 \\
\hline Hallucinogens, \% & 12.4 \\
\hline Inhalants, \% & 1.2 \\
\hline Performance-enhancing drugs, \% & 2.8 \\
\hline Barbituates, $\%$ & 8.0 \\
\hline Benzodiazepines, \% & 25.5 \\
\hline Diet pills, \% & 2.8 \\
\hline
\end{tabular}

Abbreviations: BMI, body mass index; GENOA, genes of opioid addiction; HIV, human immunodeficiency virus; SD, standard deviation.

\section{Genetic results}

Genotyping of the common SNPs of the BDNF gene (rs6265) and the $D R D 2$ gene (rs1799978) were successfully performed; the genotype call rate was excellent $(97.7 \%$ and $99.2 \%$, respectively). The minor allele frequencies were consistent with the genetic literature: 0.20 for rs6265 and 0.05 for rs 1799978 .

\section{Feasibility results}

The recruitment process yielded 260 patients from four sites during a 6 -month period, yielding a $20 \%$ recruitment rate. This was approximately equivalent to 63 patients per site, which is indicative of a feasible recruitment process. The CRFs were completed successfully for most items. The completion rates 
were: 100\% (age and sex), 99.2\% (methadone dose), 99.6\% (employment), 99.6\% (ethnicity), 94.1\% (body mass index [BMI]), and 99.6\% (alcohol use). Retention in the treatment program was on average 3.4 years, making a 12-month follow up at 3-month intervals very feasible. Finally, 99.6\% of participants provided blood samples.

\section{Discussion}

Overall, this pilot study demonstrated feasible recruitment rates, good response rates for questionnaires, significant retention in the treatment program, and very high rates of willingness to provide blood samples for DNA analysis. This assessment allows us to conclude that this study is feasible and could be expanded to include a larger sample size.

\section{Study strengths}

The strengths of this investigation include the collaborative efforts with the OATC clinical facilities and the organizational efforts brought forth by this partnership. In addition to granting investigators access to a large patient population, there is a consistent treatment strategy throughout clinics, which is an asset to any clinical study with real-world applications. As such, we believe the sample is representative of the larger population of patients undergoing methadone treatment for opioid addiction, and this supports the generalizability of our findings.

Overall, the OATC collaboration worked very well throughout the course of recruitment and completion of the pilot phase of the study, and clinics responded to queries in a timely manner, limiting the chances for missing data to arise.

\section{Key learning points}

Due to the complex nature of opioid addiction and the multiple comorbidities noted in this pilot study, the most important conclusion we were able to draw is the need for more clinically detailed assessments. We established the need for a cohort design with multiple domains to assess response to treatment since there are many factors that contribute to patient health and prognosis during treatment, in addition to continued opioid abuse. We also acknowledged the need for a detailed psychiatric assessment and reliable pain measures, after interim analyses showed high prevalence of pain and psychiatric comorbidity among the MMT patient population. Additionally, we came to realize that most of the data in our questionnaires were self-reported and thus, did not ensure accuracy, which demonstrated the need for validated tools.

Our findings also demonstrated the need for a standardized protocol to follow throughout recruitment and the data collection interviews, as well as for additional training, which would enhance accuracy of responses and minimize the number of unanswered questions.

\section{Future directions}

Preparation for the full GENOA study has begun, and we have implemented changes to the overall structure of the study. First, we have included additional measures and added validated tools to minimize recall bias and increase the accuracy of the information collected. We implemented the Maudsley Addiction Profile (MAP) instrument ${ }^{24}$ that measures the overall treatment outcome status based on several domains, including substance use, health risk behavior, physical and psychological health, and personal/social functioning. We also opted to use the full Mini International Neuropsychiatric Interview (M.I.N.I.), ${ }^{25}$ as opposed to just the drug and alcohol modules used in the pilot phase, in an effort to get an accurate depiction of patients' psychiatric condition. Additionally, our sample frequently reported chronic pain as a comorbid condition, so we included the Brief Pain Inventory $(\mathrm{BPI})^{26}$ for a more accurate assessment. This is a standardized measure that examines the overall pain status, with consideration of pain tolerance, severity, and interference with daily tasks.

Second, we have developed a study manual for clinic staff, consisting of study background and detailed instructions on CRF completion and performing the telephone training session. Although we are attempting to maximize data completion, there will inevitably be missing data, and we have decided a priori to handle this using multiple imputation.

Given the stability of number of patients per site, we expect new cases and continuation of the existing patients in each clinical site. We have initiated collaboration with six additional sites, yielding a total of ten sites out of 55. We plan to eventually expand our study to reach all 55 OATC sites. Based on the average recruitment rate, we will be able to recruit $(63 \times 55) 3,465$ participants over a 3 -year study period. This is the largest sample size for any study on methadone treatment for opioid addiction with 2,000 subjects enrolled at minimum.

Based on the high treatment retention in OATC MMT programs, we decided to take a longitudinal approach to observing these patients and have implemented a 12-month follow-up period, with interviews being conducted at 3-month intervals. This will provide us with better insight into the long-term response to treatment and will allow us to observe changes that occur as a result of treatment. 
Due to the high rate of blood collection from our sample and the efficacy of lab resources, we are now able to expand our study to include more genes of interest, which we have identified through a comprehensive literature review. In the full investigation, we plan to genotype addiction genes (OPRM1, OPRD1, BDNF, and DRD2) and metabolism genes (CYP3A4, CYP2B6, CYP2D6, ABCB1, $C E S 1$, and $C E S 2$ ) as these have potential to be involved in methadone metabolism and treatment response. Additionally, trough methadone levels will also be measured, to determine the methadone concentration in the blood as an indication of methadone metabolism rate.

\section{Limitations}

We aim to minimize selection bias by selecting individuals from clinical services where eligible patients must attend to receive treatment. We will also collect data on patients that chose not to participate and compare this with the data from our sample of participants, in an effort to address any discrepancies between the samples that may bias our findings. This will also ensure that our study maintains external validity. Use of standardized and systematic methods as well as objective measures (such as laboratory data) should minimize the occurrence of social desirability bias. Recall bias will be minimized by using a standardized instrument (MAP) designed to collect data over five time points for the past month, in combination with objective measures, thus minimizing recall bias. Loss to follow up is inevitable in any study; however, we have attempted to minimize this by choosing a feasible follow up based on duration in treatment.

This pilot study assessed the feasibility of the GENOA main study and has allowed us to conclude that our methodology and study procedures are feasible for a large full-scale study. Conducting this pilot study was an essential component to the development of the main study as it allowed us to make improvements to our research methodology and learn along the way.

\section{Acknowledgments}

We would like to sincerely thank and recognize everyone who contributed to the completion of this investigation. We thank all members of the OATC clinical staff for their great efforts in recruitment and data collection, with a special thanks going out to the recruiting nurses (D Tirabassi, T Dillon, C Geroux, B Heipel, S Rutherford, and H Cotterill). In addition, we would like to express gratitude to the McMaster University undergraduate students who volunteered a great deal of time to help with data entry and cleaning, as well as genetic analysis. These students include Sindoora Iyre, Sohail Mahmood, Leen Naji, Anuja Bhalerao, Herman Bami, and Andrew Kamphuis. We also thank all patients from the Ontario Addiction Treatment Centres (OATC) methadone treatment facilities who participated and generously donated their time, information, and samples; without them this study would not be possible. This work was supported by a Canadian Institutes of Health Research (CIHR) Drug Safety and Effectiveness Network (DSEN) grant (grant number: 126639). This work was also supported by an Innovation Award from the Department of Psychiatry and Behavioural Neurosciences, McMaster University (grant number: 2-15311).

\section{Disclosure}

The authors report no conflicts of interest in this work.

\section{References}

1. World Health Organization. The World Health Report 2002. Reducing Risks, Promoting Healthy Life. Geneva: World Health Organization; 2002. Available from: http://www.who.int/whr/2002/en/whr02 en.pdf?ua=1. Accessed June 8, 2014.

2. Ezzati M, Lopez AD, Rodgers A, Vander Hoorn S, Murray CJ; Comparative Risk Assessment Collaborating Group. Selected major risk factors and global and regional burden of disease. Lancet. 2002;360(9343): 1347-1360.

3. Zou S, Tepper M, Giulivi A. Current status of hepatitis C in Canada. Can J Public Health. 2000;91 Suppl 1:S10-S15, S10-S16.

4. Wall R, Rehm J, Fischer B, et al. Social costs of untreated opioid dependence. J Urban Health. 2000;77(4):688-722.

5. Oppenheimer E, Tobutt C, Taylor C, Andrew T. Death and survival in a cohort of heroin addicts from London clinics: a 22-year follow-up study. Addiction. 1994;89(10):1299-1308.

6. Seal KH, Kral AH, Gee L, et al. Predictors and prevention of nonfatal overdose among street-recruited injection heroin users in the San Francisco Bay Area, 1998-1999. Am J Public Health. 2001;91(11): 1842-1846.

7. Coutinho RA. HIV and hepatitis $\mathrm{C}$ among injecting drug users. $B M J$. 1998;317(7156):424-425.

8. Brooner RK, King VL, Kidorf M, Schmidt CW Jr, Bigelow GE. Psychiatric and substance use comorbidity among treatment-seeking opioid abusers. Arch Gen Psychiatry. 1997;54(1):71-80.

9. Hall W, Bell J, Carless J. Crime and drug use among applicants for methadone maintenance. Drug Alcohol Depend. 1993;31(2):123-129.

10. Okie S. A flood of opioids, a rising tide of deaths. N Engl J Med. 2010; 363(21):1981-1985.

11. Centers for Disease Control and Prevention, National Center for Health Statistics. Multiple Cause of Death 1999-2010 on CDC WONDER Online Database. 2012. Available from http://wonder.cdc.gov/mcdicd10.html. Accessed June 8, 2014.

12. Hamunen $K$, Laitinen-Parkkonen $P$, Paakkari $P$, et al. What do different databases tell about the use of opioids in seven European countries in 2002? Eur J Pain. 2008;12(6):705-715.

13. Mental Health and Addiction Information. Methadone. Toronto, ON: CAMH; 2010. Available from http://www.camh.ca/en/hospital/ health_information/a_z_mental_health_and_addiction_information/ methadone/Pages/methadone.aspx. Accessed June 30, 2014.

14. Mattick RP, Breen C, Kimber J, Davoli M. Methadone maintenance therapy versus no opioid replacement therapy for opioid dependence. Cochrane Database Syst Rev. 2009;3:CD002209. 
15. Jimenez-Treviño L, Saiz PA, García-Portilla MP, et al. A 25-year follow-up of patients admitted to methadone treatment for the first time: mortality and gender differences. Addict Behav. 2011;36(12): 1184-1190.

16. Cousins G, Teljeur C, Motterlini N, McCowan C, Dimitrov BD, Fahey T. Risk of drug-related mortality during periods of transition in methadone maintenance treatment: a cohort study. J Subst Abuse Treat. 2011;41(3):252-260.

17. Li Y, Kantelip JP, Gerritsen-van Schieveen P, Davani S. Interindividual variability of methadone response: impact of genetic polymorphism. Mol Diagn Ther. 2008;12(2):109-124.

18. Crettol S, Monnat M, Eap CB. Could pharmacogenetic data explain part of the interindividual sensitivity to methadone-induced respiratory depression? Crit Care. 2007;11(1):119.

19. Eap CB, Buclin T, Baumann P. Interindividual variability of the clinical pharmacokinetics of methadone: implications for the treatment of opioid dependence. Clin Pharmacokinet. 2002;41(14): 1153-1193.

20. Eap CB, Bertschy G, Baumann P, Finkbeiner T, Gastpar M, Scherbaum N. High interindividual variability of methadone enantiomer blood levels to dose ratios. Arch Gen Psychiatry. 1998;55(1):89-90.
21. Goldman D, Oroszi G, Ducci F. The genetics of addictions: uncovering the genes. Nat Rev Genet. 2005;6(7):521-532.

22. Amato L, Davoli M, Perucci CA, Ferri M, Faggiano F, Mattick RP. An overview of systematic reviews of the effectiveness of opiate maintenance therapies: available evidence to inform clinical practice and research. J Subst Abuse Treat. 2005;28(4):321-329.

23. Dennis BB, Bawor M, Thabane L, Sohani Z, Samaan Z. Impact of ABCB1 and CYP2B6 genetic polymorphisms on methadone metabolism, dose and treatment response in patients with opioid addiction: a systematic review and meta-analysis. PLoS One. 2014;9(1):e86114.

24. Marsden J, Gossop M, Stewart D, et al. The Maudsley Addiction Profile (MAP): a brief instrument for assessing treatment outcome. Addiction. 1998;93(12):1857-1867.

25. Sheehan DV, Lecrubier Y, Sheehan KH, et al. The Mini-International Neuropsychiatric Interview (M.I.N.I.): the development and validation of a structured diagnostic psychiatric interview for DSM-IV and ICD-10. J Clin Psychiatry. 1998;59 Supp1 20:S22-S33; quiz S34-S57.

26. Cleeland CS, Ryan KM. Pain assessment: global use of the Brief Pain Inventory. Ann Acad Med Singapore. 1994;23(2):129-138.
Neuropsychiatric Disease and Treatment

\section{Publish your work in this journal}

Neuropsychiatric Disease and Treatment is an international, peerreviewed journal of clinical therapeutics and pharmacology focusing on concise rapid reporting of clinical or pre-clinical studies on a range of neuropsychiatric and neurological disorders. This journal is indexed on PubMed Central, the 'PsycINFO' database and CAS,

\section{Dovepress}

and is the official journal of The International Neuropsychiatric Association (INA). The manuscript management system is completely online and includes a very quick and fair peer-review system, which is all easy to use. Visit http://www.dovepress.com/testimonials.php to read real quotes from published authors. 